\title{
Morphology, Molecular Identity, and Pathogenicity of Verticillium dahliae and V. longisporum Associated with Internally Discolored Horseradish Roots
}

Jun Myoung Yu, Department of Plant Pathology and Microbiology, Texas A\&M University, College Station 77843-2133; Ibrahim H. Cafarov, Department of Plant Protection, Azerbaijan State Agricultural University, Ganja AZ 2000, Azerbaijan; and Mohammad Babadoost, Department of Crop Sciences, University of Illinois, Urbana 61801

\begin{abstract}
Yu, J. M, Cafarov, I. H., and Babadoost, M. 2016. Morphology, molecular identity, and pathogenicity of Verticillium dahliae and V. longisporum associated with internally discolored horseradish roots. Plant Dis. 100:749-757.

During 2008 to 2009, 255 isolates of Verticillium were obtained from internally discolored horseradish roots collected from California, Illinois, and Ontario. Twenty representative isolates were selected according to morphological features and geographic origin for further characterization. Based on the conidial size, the isolates were divided into two groups: Verticillium dahliae $(4.4 \pm 1.23 \mu \mathrm{m})$ and $V$. longisporum $(7.8 \pm$ $1.76 \mu \mathrm{m}$ ). Genetic diversity of the isolates was determined by sequence analysis of the internal transcribed spacer (ITS) and two mitochondrial genes (cytochrome oxidase subunit III [cox3] and NADH dehydrogenase subunit I [nad1]). Based on ITS analysis, Verticillium isolates were

divided into two clades: $V$. dahliae and $V$. longisporum. However five isolates of $V$. longisporum (identified based on conidial size) were clustered with a $V$. dahliae clade, whereas the other five isolates formed a distinct $V$. longisporum clade. Combined analysis of the mitochondrial genes cox3 and nadl showed that the two genetic clades of $V$. longisporum in ITS region analysis corresponded to the previously reported $V$. longisporum lineage A1/D3 and A1/D2. Pathogenicity tests revealed that all tested Verticillium isolates caused internal discoloration of horseradish roots, and there were no significant differences in either incidence or severity of root discoloration among the genetic groups.
\end{abstract}

Horseradish (Armoracis rusticana Gaertn., Mey. \& Scherb.) is a member of the Brassicaceae family and is grown for its white, fleshy, and pungent roots, which are used primarily as a condiment (Babadoost et al. 2004; Courter and Rhodes 1969). The major areas of commercial cultivation of horseradish in North America are California, Illinois, and Wisconsin in the United States and Ontario in Canada (Babadoost et al. 2004; Yu and Babadoost 2013). Approximately $50 \%$ of the total horseradish crop in the United States is grown in the Mississippi River Valley, near East St. Louis, IL (Babadoost et al. 2004; Rhodes 1977; Yu and Babadoost 2013). Eau Claire, WI and Tule Lake, CA are two other major horseradish production areas in the United States (Babadoost et al. 2004; Yu and Babadoost 2013).

Internal discoloration of roots occurs worldwide and affects production of horseradish in Illinois as well as other areas (Babadoost et al. 2004; Eastburn and Chang 1994; Potschke 1923; Yu and Babadoost 2013). The disease begins with dark-brown to black discoloration of the vascular system and gradually spreads inward (core) and outward (cortex) (Babadoost et al. 2004; Eastburn and Chang 1994; Yu and Babadoost 2013). Internally discolored horseradish roots are not acceptable for processing and, thus, are discarded (Babadoost et al. 2004; Eastburn and Chang 1994).

Internal root discoloration of horseradish was first observed in Germany in 1860s and was reported by Sorauer (1899). In the United States, internal discoloration of horseradish was first described in 1931 in Michigan, where $20 \%$ of the yield was lost due to root infection (Boning 1938). Since the early 1980s, horseradish producers in Illinois have experienced substantial reductions in marketable yield of horseradish due to discoloration and rotting of roots (Atibalentja and Eastburn 1998; Babadoost et al. 2004; Eastburn and Chang 1994). Yield losses of up to $100 \%$ due to the internal root discoloration have frequently been reported in Illinois (Babadoost et al. 2004; Yu and Babadoost 2013).

Corresponding author: M. Babadoost; E-mail: babadoos@illinois.edu

Accepted for publication 12 October 2015.

http://dx.doi.org/10.1094/PDIS-08-15-0846-RE

(C) 2016 The American Phytopathological Society
A species of Verticillium Nees was reported to be associated with the internally discolored roots of horseradish (Potschke 1923), which was identified as Verticillium dahliae Kleb. in both Europe and the United States (Korff and Boning 1934; Mueller et al. 1982). Mueller et al. (1982) reported $V$. dahliae as the primary agent associated with internally discolored horseradish roots in Wisconsin. However, Percich and Johnson (1990) isolated V. dahliae, Fusarium roseum 'Acuminatum', and Pseudomonas fluorescens from internally discolored horseradish roots in Wisconsin and reported the disease as a root rot complex of horseradish. Further, Babadoost et al. (2004) reported that internal discoloration of horseradish root is a disease complex caused by at least three fungal species: $V$. dahliae, $V$. longisporum, and $F$. solani. Finally, $\mathrm{Yu}$ and Babadoost (2013) reported that $F$. commune and $F$. oxysporum also cause internal root discoloration of horseradish.

$V$. dahliae is a well-known soilborne pathogen that causes wilt in a broad range of plant species, including brassicas (Isaac 1957; Koike et al. 1994; Svensson and Lerenius 1987). In 1961, a variant of $V$. dahliae from horseradish was described by Stark that produced conidia approximately twice as long as those of typical $V$. dahliae strains. This variant was named $V$. dahliae var. longisporum. Based on distinct morphological, molecular, and virulence characteristics, Karapapa et al. (1997) suggested that this fungus should be classified as a new species, $V$. longisporum. In addition, several researchers have demonstrated molecular differences between $V$. longisporum and $V$. dahliae isolates that support recognition of the newly erected species (Fahleson et al. 2004; Pantou et al. 2005; Zeise and von Tiedemann 2001). However, considerable intraspecific diversity has been identified within isolates of $V$. longisporum, giving rise to a controversy regarding the proper taxonomic status of $V$. longisporum versus V. dahliae var. longisporum (Barbara and Clewes 2003; ColladoRomero et al. 2010; Collins et al. 2005; Karapapa et al. 1997; Pantou et al. 2005; Zeise and von Tiedemann 2001). Thus, some researchers have questioned the recognition of the new species because of less homogeneity among isolates of the group (Clewes et al. 2008; Collins et al. 2003, 2005; Klosterman et al. 2009).

Recent molecular analyses have suggested that $V$. longisporum is the result of hybridization between $V$. dahliae and $V$. albo-atrum (Clewes et al. 2008; Collins et al. 2003). Based on the phylogenetic analyses of seven nuclear loci and a dataset of 203 isolates of $V$. longisporum, V. dahliae, and related species, Inderbitzin et al. (2011a,b) 
reported that $V$. longisporum is an allodiploid hybrid that originated at three separate times from four different lineages representing three different parental species: species A1, species D1, and V. dahliae.

Although genetic diversity within and among Verticillium spp. from several host species has been elucidated using molecular genetic techniques (Carder and Barbara 1994; Clewes et al. 2008; Inderbitzin et al. 2011a,b; Klosterman et al. 2009; Pantou et al. 2005; Steventon et al. 2002), comprehensive studies on the genetic diversity within and among Verticillium spp. from horseradish have not been conducted. The objective of this study was to characterize Verticillium spp. associated with horseradish roots showing internal discoloration in California, Illinois, and Ontario using molecular phylogenetic analysis along with morphological grouping and their pathogenicity.

\section{Materials and Methods}

Root samples. In total, 496 internally discolored horseradish roots, including 75, 306, and 115 roots from commercial fields in California, Illinois, and Ontario, respectively, were collected. Each root was washed and sectioned at one-third length from the top and one-third from bottom of the root. Roots with visible discoloration at both cross sections were selected for isolating pathogens. Selected roots were tested immediately for the presence of pathogens or stored at $4{ }^{\circ} \mathrm{C}$ until they were tested within 2 weeks.

Isolation of Verticillium. Fungal isolation was carried out as previously described by Babadoost et al. (2004). A root segment was cut

Table 1. Frequency of isolation of Verticillium dahliae and $V$. longisporum from roots of horseradish cultivars grown in California, Illinois, and Ontario in 2008 and 2009

\begin{tabular}{llrcc}
\hline & & & \multicolumn{2}{c}{ Isolation frequency } \\
\cline { 5 - 5 } Location & Cultivar & $N^{\mathbf{z}}$ & V. dahliae & V. longisporum \\
\hline California & Czechoslovakia & 45 & 2 & 19 \\
& Tule Lake & 30 & 0 & 15 \\
Illinois & Big Top Western & 72 & 39 & 8 \\
& 1573 & 234 & 71 & 18 \\
Ontario & 1590 & 115 & 77 & 6 \\
\hline
\end{tabular}

${ }^{y}$ Identification of the species was based on the morphological characteristics of conidia, conidiophores, and microsclerotia of the isolate.

${ }^{\mathrm{z}}$ Number of roots assayed. from the middle of each collected symptomatic root. The outer layers (approximately $1 \mathrm{~mm}$ ) of the segments were peeled to remove fungal and bacterial contaminants on the root surface. The peeled root segments were surface sterilized by immersing roots in a $6 \% \mathrm{NaClO}$ (full-strength commercial bleach) solution for $1 \mathrm{~min}$, followed by dipping in $95 \%$ ethanol for $3 \mathrm{~min}$ and rinsing in sterile-distilled water (SDW) three times, each time for $1 \mathrm{~min}$. The segment was then blotted with sterilized blotter paper and cut into $3-$ to 5 -mm pieces. Five pieces from each root were placed onto potato dextrose agar (PDA; Difco, Sparks, MD) with $4 \mathrm{ml}$ of $25 \%$ lactic acid in 1,000 ml of culture medium (A-PDA) in petri plates. The plates were then incubated at 22 to $24^{\circ} \mathrm{C}$ in the dark for 15 days. The plates were examined 5, 10, and 15 days after incubation, and developing fungal colonies were transferred aseptically onto PDA in petri plates. The cultures were incubated under a cycle of $12 \mathrm{~h}$ of near-UV light and $12 \mathrm{~h}$ of darkness per day for 8 to 10 days and growing colonies were identified following their sporulation. In total, 255 Verticillium colonies (each representing one root) were subcultured onto PDA in petri plates until pure cultures were obtained. Based on the morphological features and geographic origin (Table 1), 20 isolates were selected for further studies. Single conidial cultures were prepared from these 20 isolates (Babadoost et al. 2004) and maintained as stock cultures on PDA slants in the dark at $4^{\circ} \mathrm{C}$. The reference isolates examined are listed in Table 2 with their National Center for Biotechnology Information GenBank accession numbers.

Morphological characteristics for identification of the isolates. For morphological observations, in total, 20 isolates of Verticillium (Table 2) were grown on PDA in petri plates at $25^{\circ} \mathrm{C}$ in the dark for 20 days. The isolates were identified based on morphological characteristics of conidia and microsclerotia, as described by the previous investigators (Hawksworth and Talboys 1970; Karapapa et al. 1997; Steventon et al. 2002). For conidia, spore suspensions in SDW were prepared from cultures after 7 days and the lengths of 30 conidia from each isolate were measured. Morphological characteristics of microsclerotia of all isolates were examined using light microscopy after growing them on PDA in petri plates for 14 days.

DNA extraction. A small plaque of each isolate was transferred into $150 \mathrm{ml}$ of potato dextrose broth (PDB; Difco) in a 250-ml flask and the flask was placed onto a shaking incubator $(150 \mathrm{rpm})$ at $25^{\circ} \mathrm{C}$ for 7 to 10 days. Fungal mycelia were recovered from the PDB culture by centrifugation and filtration through sterilized filter paper (Whatman, Maidston, UK). Total DNA was extracted from fungal

Table 2. Geographic origin (Origin), year of isolation (Year), conidial length (Length; $n=30$ ), and GenBank accession numbers for internal transcribed spacer (ITS), cytochrome oxidase subunit III (cox3), and NADH dehydrogenase subunit I (nad1) sequences of Verticillium isolates used in this study

\begin{tabular}{|c|c|c|c|c|c|c|c|}
\hline \multirow[b]{2}{*}{ Species } & \multirow[b]{2}{*}{ Isolate code } & \multirow[b]{2}{*}{ Origin } & \multirow[b]{2}{*}{ Year } & \multirow[b]{2}{*}{ Length $(\boldsymbol{\mu m})^{\mathrm{z}}$} & \multicolumn{3}{|c|}{ GenBank accession numbers } \\
\hline & & & & & ITS & $\cos 3$ & nad1 \\
\hline Verticillium dahliae & CAV1003 & Ontario & 2008 & $4.8(1.34)$ & KC834724 & KC852087 & KC852100 \\
\hline V. dahliae & CAV1004 & Ontario & 2008 & $5.0(1.47)$ & $\mathrm{KC} 834725$ & KC852088 & KC852101 \\
\hline V. dahliae & CBV1006 & Ontario & 2008 & $3.6(0.86)$ & KC834726 & KC852089 & KC852102 \\
\hline V. dahliae & CBV1014 & Ontario & 2008 & $4.7(1.28)$ & KC834727 & KC852090 & KC852103 \\
\hline V. dahliae & CBV1046 & Ontario & 2008 & $3.5(1.16)$ & $\mathrm{KC} 834728$ & KC852091 & HM988737 \\
\hline V. dahliae & SCV 1005 & Illinois & 2008 & $5.0(1.28)$ & KC834729 & KC852092 & KC852104 \\
\hline V. dahliae & SCV1010 & Illinois & 2008 & $3.6(0.73)$ & KC834730 & HM804952 & HM988738 \\
\hline V. dahliae & SDV1002 & Illinois & 2008 & $4.8(1.34)$ & KC834731 & HM804953 & HM988736 \\
\hline V. dahliae & SDV1006 & Illinois & 2008 & $5.2(1.54)$ & KC834732 & KC852093 & KC852105 \\
\hline V. dahliae & SDV1025 & Illinois & 2008 & $4.3(1.33)$ & KC834733 & KC852094 & KC852106 \\
\hline V. longisporum & CBV2034 & Ontario & 2008 & $8.5(2.18)$ & KC834714 & HM988730 & HM988739 \\
\hline V. longisporum & SCV2008 & Illinois & 2008 & $8.0(2.12)$ & $\mathrm{KC} 834715$ & KC852082 & KC852095 \\
\hline$V$. longisporum & SDV2041 & Illinois & 2008 & $7.4(1.48)$ & KC834716 & KC852083 & KC852096 \\
\hline V. longisporum & SDV2064 & Illinois & 2008 & $8.5(2.09)$ & $\mathrm{KC} 834717$ & HM988732 & HM988740 \\
\hline V. longisporum & SDV2066 & Illinois & 2008 & $8.3(2.19)$ & KC834718 & KC852084 & KC852097 \\
\hline V. longisporum & TAV3001 & California & 2009 & $7.0(1.39)$ & KC834719 & HM988734 & HM988743 \\
\hline V. longisporum & TBV3014 & California & 2009 & $6.7(1.51)$ & KC834720 & HM988733 & HM988742 \\
\hline V. longisporum & VAV3003 & California & 2009 & $7.1(1.79)$ & KC834721 & HM988735 & HM988744 \\
\hline V. longisporum & VAV3021 & California & 2009 & $6.8(1.54)$ & KC834722 & KC852085 & KC852098 \\
\hline V. longisporum & VBV3004 & California & 2009 & $6.7(1.26)$ & KC834723 & KC852086 & KC852099 \\
\hline
\end{tabular}

$\mathrm{z}$ Values in parentheses are the standard deviations of measurements of conidial length. 
mycelia using a Fast DNA Spin kit (MP Bio, Solon, OH), according to the manufacturer's recommended protocol.

Polymerase chain reaction test. A polymerase chain reaction (PCR) test was performed to amplify the internal transcribed spacer (ITS) region (ITS1-5.8S-ITS2) using the primer pair ITS5/ITS4 (GGA AGTAAAAGTCGTAACAAGG/TCCTCCGCTTATTGATATGC) (White et al. 1990). Also, two mitochondrial genes, cytochrome oxidase subunit III ( $\operatorname{cox} 3$ ) and NADH dehydrogenase subunit I (nad 1), were amplified using primer pairs $\operatorname{cox} 3 \mathrm{~A} / \operatorname{cox} 3 \mathrm{~B}$ (TGATTTAGAGATST AATATCAGAAG/CCGTGGAAACCTGTSCCAAAATA) and nad1A/nad1B (ATGGCSAGTATGCAAAGAAGA/GCATGTTC TGTCATAAASCCACT AAC), respectively, following methods developed by Kouvelis et al. (2004) and Pantou et al. (2003). A PCR mixture with a total volume of $50 \mu \mathrm{l}$ was prepared, which contained $10 \times$ PCR buffer, $2.0 \mathrm{mM} \mathrm{MgCl} 2,2.5 \mathrm{mM}$ dNTP, $10 \mu \mathrm{M}$ each primer, $30 \mathrm{ng}$ of $\mathrm{Taq}$ polymerase (Promega Corp., Madison, WI), and $2 \mu 1$ of DNA extract. PCR conditions for ITS were initial denaturation at $95^{\circ} \mathrm{C}$ for $2 \mathrm{~min} ; 35$ cycles of denaturation at $94^{\circ} \mathrm{C}$ for $45 \mathrm{~s}$, annealing at $54^{\circ} \mathrm{C}$ for $1 \mathrm{~min}$, and extension at $72^{\circ} \mathrm{C}$ for $1.5 \mathrm{~min}$; followed by a final extension at $72^{\circ} \mathrm{C}$ for $5 \mathrm{~min}$. The PCR cycles for the two mitochondrial genes were initial denaturation at $94^{\circ} \mathrm{C}$ for $3 \mathrm{~min} ; 30$ cycles of denaturing at $94^{\circ} \mathrm{C}$ for $1 \mathrm{~min}$, annealing at $48^{\circ} \mathrm{C}$ for $\operatorname{cox} 3$ primers and $50^{\circ} \mathrm{C}$ for $n a d 1$ primers for $1 \mathrm{~min}$, and extension at $74^{\circ} \mathrm{C}$ for $2 \mathrm{~min}$; and final extension at $74^{\circ} \mathrm{C}$ for $5 \mathrm{~min}$.

PCR was conducted using a PCT-200 thermal cycler (MJ Research Inc., Waltham, MA). All PCR amplifications were verified by electrophoresis with $10 \mu \mathrm{l}$ of each PCR product in a $1 \%$ agarose gel with $1 \times$ Tris-acetate-EDTA buffer stained with ethidium bromide. Gels were visualized and documented under UV light.

DNA sequencing and phylogenetic analyses. PCR products were purified using the Wizard SV gel and PCR Clean-Up system (Promega Corp.), according to the manufacturer's protocol. Purified PCR products were sequenced using an automated sequencer at the Core DNA Sequencing Facility of the University of Illinois at Urbana-Champaign. Sequencing was performed using the same forward and reverse primer sets that were used for PCR amplification.

The results of the sequence were first edited using Clustal $\mathrm{X}$ (Thompson et al. 1997) and BioEdit version 7.0.9 (Hall 1999), after which the alignment was refined using PHYDIT, version 3.2 (Jeon et al. 2005). All sequences used for phylogenetic analyses were deposited into GenBank (Table 2).
Phylogenetic analyses were performed on PAUP 4.0 10b (Swofford 1998). Ambiguously aligned regions and sequence gaps were treated as missing data and, thus, excluded from analyses. Maximum-parsimony (MP) analysis was estimated using heuristic searches consisting of random addition order and tree bisection-reconnection branch swapping. Maximum-likelihood (ML) analyses were performed with RAxML 7.2.8 HPC BlackBox (Stamatakis. 2006; Stamatakis et al. 2008) using the GTRGAMMA model of nucleotide substitution. Mega v 5.05 (Tamura et al. 2011) was used for editing of the obtained trees. Trees were rooted with Gibellulopsis nigriscens used as the outgroup. Parsimony and ML bootstrapping were performed with 1,000 replicates to assess the relative stability of the branches. The information of the reference strains was obtained from the National Center for Biotechnology Information (NCBI) GenBank database (http://www.ncbi.nlm.nih.gov/) and listed in Table 3.

Pathogenicity tests. Pathogenicity tests of the isolates were conducted as previously described (Babadoost et al. 2004; Kouvelis et al. 2004). Two horseradish cultivars, '1573' and 'Big Top Western' (BTW), which are susceptible to internal root discoloration (Babadoost et al. 2004; Yu and Babadoost 2013), and two isolates of each species or group of Verticillium were used for the pathogenicity tests.

Asymptomatic horseradish roots were collected from commercial fields and the roots were treated at $47^{\circ} \mathrm{C}$ for $20 \mathrm{~min}$ prior to inoculation to eliminate the possible set-borne inoculum (inoculum carried in symptomatic and asymptomatic propagative roots; Eranthodi et al. 2010). Treated roots were then planted in pots ( 50 by 35 by $10 \mathrm{~cm}$ ) containing a pasteurized mix of soil-peat-perlite (1:1:1) (Babadoost et al. 2004). The pots were placed in a greenhouse at 20 to $26^{\circ} \mathrm{C}$. After 30 days, plants were removed from the pots, soil was gently cleaned from the main root and lateral roots, and the roots were inoculated with one isolate of $V$. dahliae and two isolates of $V$. longisporum separately and also combined together. The experiment was repeated once.

To produce Verticillium inoculum, 2-week-old cultures of Verticillium spp. on PDA in petri plates were macerated with $15 \mathrm{ml}$ of SDW. The conidial suspension (1 ml) was spread onto the surface of PDA in each petri plate. The inoculated plates were then incubated at 22 to $24^{\circ} \mathrm{C}$ under a cycle of $12 \mathrm{~h}$ of fluorescent light and $12 \mathrm{~h}$ of darkness per day for 3 weeks. The cultures were then blended with $200 \mathrm{ml}$ of distilled water (DW) at high speed for $30 \mathrm{~s}$. The resulting suspension was passed through a series of 500-, 180-, and 38- $\mu \mathrm{m}$ sieves. Material (microsclerotia) caught on the $38-\mu \mathrm{m}$ sieve was rinsed with DW

Table 3. Reference strains of Verticillium spp. and Gibellulopsis nigrescens (with their GenBank accession numbers) used for phylogenetic analyses

\begin{tabular}{|c|c|c|c|c|c|c|}
\hline \multirow[b]{2}{*}{ Species } & \multirow[b]{2}{*}{ Isolate code } & \multirow[b]{2}{*}{ Host, substrate } & \multirow[b]{2}{*}{ Origin $^{z}$} & \multicolumn{3}{|c|}{ GenBank accession numbers ${ }^{y}$} \\
\hline & & & & ITS & $\cos 3$ & nad1 \\
\hline Verticillium dahliae & 76 & Cotton & Greece & - & AY555919 & AY555975 \\
\hline V. dahliae & M5 & Egg plant & Moldova & AY555948 & AY555921 & AY555977 \\
\hline V. dahliae & PD332 & Lettuce & California & HQ206718 & - & - \\
\hline V. dahliae & PD727 & Horseradish & Illinois & HQ206917 & - & - \\
\hline V. dahliae & PD728 & Horseradish & Illinois & HQ206918 & - & - \\
\hline V. dahliae & UZ132 & Cotton & Uzbekistan & AY555949 & AY555922 & AY555978 \\
\hline$V$. longisporum & G19 & Oilseed rape & Germany & AY555950 & AY555923 & AY555979 \\
\hline$V$. longisporum & $\mathrm{K} 2$ & Oilseed rape & Russia & AY555951 & AY555924 & AY555980 \\
\hline$V$. longisporum & $\mathrm{K} 12$ & Oilseed ape & Russia & AY555952 & AY555925 & AY555981 \\
\hline$V$. longisporum & PD687 & Horseradish & German & HQ206893 & - & - \\
\hline$V$. longisporum & PD730 & Horseradish & Illinois & HQ206920 & - & - \\
\hline V. albo-atrum & 1776 & Hop & United Kingdom & AY555953 & AY555926 & AY555982 \\
\hline V. albo-atrum & V90 & Cotton & Middle East & AY555954 & AY555927 & AY555983 \\
\hline V. albo-atrum & PD670 & Potato & Wisconsin & JN187990 & - & - \\
\hline V. albo-atrum & PD748 & Soil & Canada & JN188017 & - & - \\
\hline V. klebahnii & PD347 & Artichoke & California & JN187965 & - & - \\
\hline V. nubilum & IMI278734 & Potato & United Kingdom & AY935948 & - & - \\
\hline V. tricorpus & PD593 & Potato & Japan & JN187974 & - & - \\
\hline V. tricorpus & PD685 & Larkspur & Japan & JN187992 & - & - \\
\hline G. nigrescens & 193 & Unknown & Unknown & AY555959 & AY555932 & AY555987 \\
\hline$G$, nigrescens & PD595 & Anemone & Japan & JN187976 & - & - \\
\hline
\end{tabular}

y ITS = internal transcribed spacer, cox3 = cytochrome oxidase subunit III, nad 1 = NADH dehydrogenase subunit I, and $-=$ not available.

${ }^{\mathrm{z}}$ Geographic origin in countries, regions, and the states of California and Illinois in the United States. 
and the collected microsclerotia were resuspended in $0.5 \%$ sterilized agar solution to prepare sticky inoculums (Babadoost et al. 2004). An inoculum suspension of 500 microsclerotia/ml was prepared using a spore-counting chamber.

Roots of plants grown in the greenhouse were dipped in the $0.5 \%$ agar suspension of microsclerotia for $15 \mathrm{~s}$, after which the inoculated plants were planted in plastic pots containing the pasteurized soilpeat-perlite (1:1:1) mix. Roots of control plants were dipped in sterilized $0.5 \%$ agar solution. Pots were placed in the greenhouse at 20 to $26^{\circ} \mathrm{C}$ under a combination of 1,000-W high-pressure sodium and mercury vapor lamps with a photoperiod of 12-h/day. Each inoculation treatment included four replications, each with six plants, in a randomized complete block design.

Plants were removed at 4-week intervals until 4 months after inoculation. Roots were washed, cut, and evaluated for both incidence and severity of internal discoloration in the cross-section of the middle of the root. Severity of root discoloration (percent area of root at cross section discolored) was assessed using a 0 -to-4 scale, where $0=$ $0,1=1$ to 10 (average 5), $2=11$ to 25 (17.5), $3=26$ to 50 (37.5), and $4=51$ to $100 \%(75 \%)$. Root sections were surface sterilized by soaking in $6 \% \mathrm{NaClO}$ solution for $1 \mathrm{~min}$, dipping in $95 \%$ ethanol for $2 \mathrm{~min}$, and rinsing in SDW three times, each time for $1 \mathrm{~min}$. Sections ( $5 \mathrm{~mm}$ thick) of roots were cut and placed on A-PDA in petri plates for reisolation of the pathogens. Plates were incubated at 22 to $24^{\circ} \mathrm{C}$ under a cycle of $12 \mathrm{~h}$ of fluorescent light and $12 \mathrm{~h}$ of darkness per day. Growing fungal colonies were transferred onto PDA, after which the species were identified base on morphology of their conidia and conidiophores. The pathogenicity test was repeated once. Statistical analyses of the data were performed using the analysis of variance procedure of SAS (version 9.4; SAS Institute, Cary, NC).

\section{Results}

Isolated fungi. Fungi were isolated from 421 of 496 roots, which included 65 of 75, 250 of 306, and 106 of 115 of roots collected from California, Illinois, and Ontario, respectively (Table 1). In total, 586 fungal isolates comprising 106, 288, and 192 isolates from California, Illinois, and Ontario, respectively, were collected. Up to three different fungi were isolated from some of the roots. The majority of the isolates were Verticillium (44\% of the total isolates) and Fusarium (25\% of the total isolates) species based on the morphological characteristics and ITS sequence analysis. Verticillium spp. were isolated from 34, 47, and $43 \%$ of roots collected from California, Illinois, and Ontario, respectively. In total, 255 isolates were identified as Verticillium.

Morphological identification of Verticillium isolates. Based on conidial length and microsclerotial shape, Verticillium isolates were divided into two groups. One group produced a white colony with abundant fluffy mycelium on PDA. The colony was creamy white initially but gradually became dark and densely compacted and appressed to agar. Abundant conidia were produced by the colonies, which were hyaline, cylindrical to oval, measuring $<6.0 \mu \mathrm{m}$ in length $(4.4 \pm 1.23 \mu \mathrm{m})$ (Fig. 1A). Conidiophores were hyaline, verticillately branched, and had 2 to 4 phialides per node (Fig. 1B). Microsclerotia were dark brown to black, spherical and compact, immersed in agar, measuring 20 to $100 \mu \mathrm{m}$ in diameter (Fig. 1C). Aggregates of microsclerotia up to $200 \mu \mathrm{m}$ in diameter developed. These characteristics fit the description of $V$. dahlia (Collins et al. 2003; Hawksworth and Talboys 1970; Karapapa et al. 1997). Ten isolates, including CAV1003, CAV1003, CAV1004, CBV1006, CBV1014, CBV1046, SCV1005, SCV1010, SDV1002, SDV1006, and SDV1025, belonged to this group.

Isolates that belonged to another group produced whitish to gray colonies, with sparse aerial mycelium on PDA. Conidia produced by the colonies were hyaline, cylindrical to ellipsoid, measuring $>6.5 \mu \mathrm{m}$ in length $(7.8 \pm 1.76 \mu \mathrm{m})$ (Fig. 1D). Conidiophores were hyaline and had 2 to 4 (mostly 3 ) phialides per node (Fig. 1E). Microsclerotia were variable in shape (elongate to irregularly spherical) and variable in size (30 to $250 \mu \mathrm{m}$ in diameter), with aggregates of microsclerotia measuring $\geq 300 \mu \mathrm{m}$ in diameter (Fig. 1F). These characteristics fit the description of $V$. longisporum (Collins et al. 2003; Karapapa et al. 1997). Ten isolates, including CBV2034, SCV2008, SDV2041, SDV2064, SDV2066, TAV3001, TBV3014, VAV3003, VAV3021, and VBV3004, belonged to this group.

Molecular characteristics of Verticillium isolates. The sequences from PCR products of ITS regions were analyzed with retrieved sequences from GenBank. Sequences were deposited in GenBank under accession numbers KC834714 to KC834733 (Table 2). Amplification of the ITS regions of the Verticillium isolates generated 480-bp fragments, and alignment of the sequences resulted in a dataset of 468 characters. Based on 25 parsimonyinformative characters, the three most parsimonious trees were located with a tree length of 82 steps (consistency index $(\mathrm{CI})=$ 0.9268 , retention index $(\mathrm{RI})=0.9492$, homoplasy index $(\mathrm{HI})=$ 0.0732 , released consistency index $(R C)=0.8801)$. ML analysis produced a tree with a log likelihood (In) of -1034.035568 (Fig. 2).

Based on phylogenetic analysis of the ITS region, the Verticillium isolates from horseradish were ambiguously divided into two clades, $V$. dahliae and $V$. longisporum. All 10 isolates of $V$. dahliae (CAV1003, CAV1004, CBV1006, CBV1014, CBV1046, SCV1005, SCV1010, SDV1002, SDV1006, and SDV1025), identified based on their morphological characteristics, fell into one clade group. Five isolates (TAV3001, TBV3014, VAV3003, VAV3021, and VBV3004), which were identified as V. longisporum, were also clustered into the $V$. dahliae clade along with reference strains of

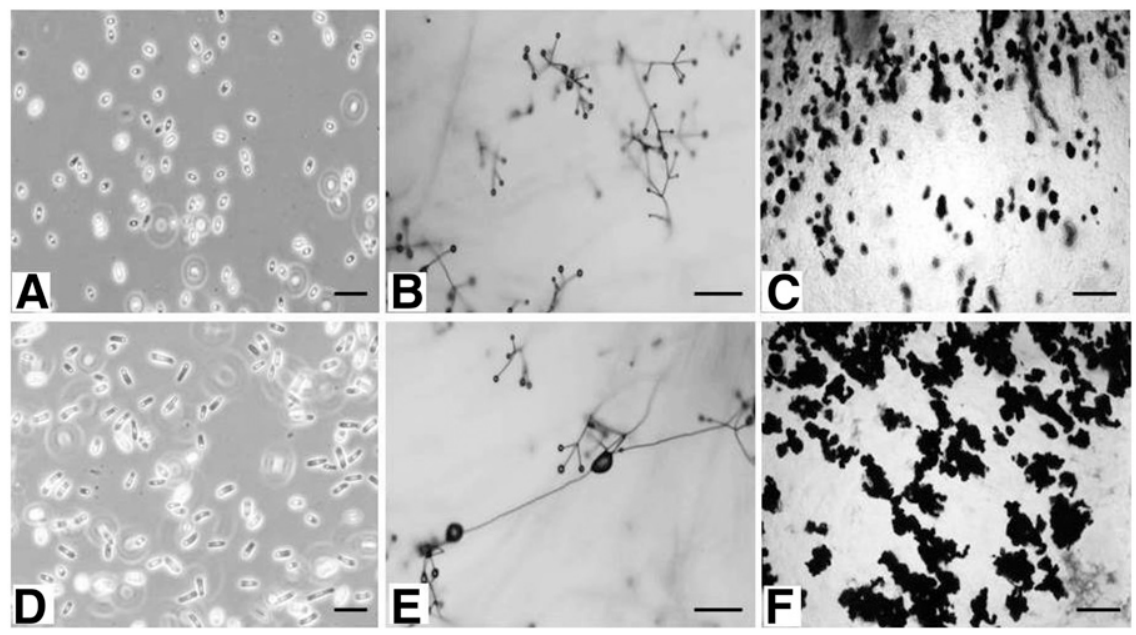

Fig. 1. Morphological characteristics of $\mathbf{A}$ to $\mathbf{C}$, Verticillium dahliae and $\mathbf{D}$ to $\mathbf{F}, \mathrm{V}$. longisporum grown on potato dextrose agar. $\mathbf{A}$ and $\mathbf{D}$, conidia; $\mathbf{B}$ and $\mathbf{E}$, conidiophores; and $\mathbf{C}$ and $\mathbf{F}$, microsclerotia. Scale bars: $A$ and $D=20 \mu \mathrm{m}, B$ and $E=20 \mu \mathrm{m}$, and $C$ and $F=300 \mu \mathrm{m}$. 
V. dahliae (strains D322, M5, PD727, PD728, and UZ132) and reference strains of $V$. longisporum (strains G19 and PD687) with strong bootstrap percentage (BP) support (96\% ML BP, 97\% MP BP). The other five isolates (CBV2034, SCV2008, SDV2041, SDV2064, and SDV2066), identified as $V$. longisporum, and reference strains of $V$. longisporum (K2 and K12) were clustered together into a separate clade with bootstrap support of 100\% ML and 62\% MP (Fig. 2). Overall, the results showed that the 10 isolates identified as $V$. longisporum based on morphological characteristics were divided into two groups. One group (group A: five isolates from California) formed a monophyletic clade with all isolates identified as $V$. dahliae based on morphological characteristics. ITS sequences of these isolates and reference strain $V$. longisporum PD687 were identical to those of most $V$. dahliae isolates. The other group (group B), including four isolates from Illinois and one isolate from Ontario, formed a distinct clade (Fig. 2).
The sequences of the PCR products of $\operatorname{cox} 3$ were analyzed with sequences retrieved from GenBank. Sequences of the products were deposited in GenBank under accession numbers KC852082 to KC852094 and HM804952 to HM988735 (Table 2). Amplification of the cox3 gene generated 380-bp fragments, and alignment of the sequences resulted in a dataset of 364 characters. Based on 32 parsimony-informative characters, the three most parsimonious trees were generated with a tree length of 84 steps $(\mathrm{CI}=0.9167, \mathrm{RI}=$ $0.9014, \mathrm{HI}=0.0833, \mathrm{RC}=0.8263)$. The sequences of the PCR products of nadl were also analyzed with sequences retrieved from GenBank. Sequences were deposited in GenBank under accession numbers KC852095 to KC852106 and HM988735 to HM988744 (Table 2). Amplification of the nad1 gene generated 500-bp fragments, and alignment of the sequences resulted in a dataset of 484 characters. Based on 39 parsimony-informative characters, the two most parsimonious trees generated were with a tree length of 157 steps

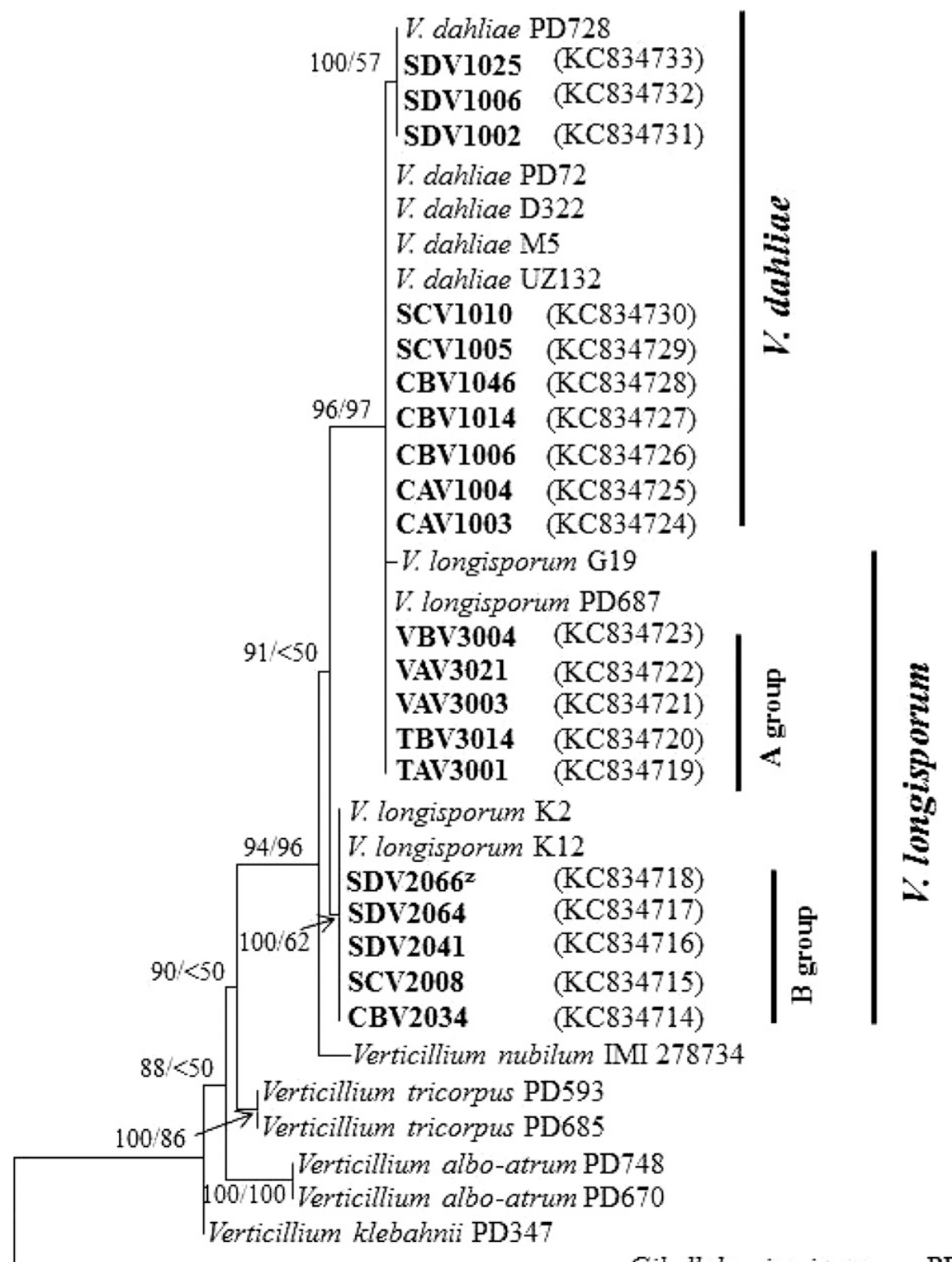

$\overline{0.02}$

Fig. 2. Maximum-likelihood (ML) tree based on internal transcribed spacer sequence data of the examined 20 isolates. ML and maximum-parsimony bootstrap values $\geq 50 \%$ are shown at nodes. Negative log likelihood of the ML tree $=-1,034.035568$. Outgroup: Gibellulopsis nigrescens. Verticillium longisporum isolates in group A were from California, and $V$. longisporum isolates in group B and V. dahliae isolates were from Illinois and Ontario. V. Iongisporum isolate SDV-2066 $\left({ }^{\mathrm{Z}}\right)$ is the same isolate as reference strain $\mathrm{V}$. longisporum PD730, which also was reported by Inderbitzin et al. (2011b). 
$(\mathrm{CI}=0.8726, \mathrm{RI}=0.8148, \mathrm{HI}=0.1274, \mathrm{RC}=0.7110)$. The combined sequence data for cox 3 and nad 1 resulted in a dataset of 848 characters, with 71 parsimony-informative characters. For MP analysis, the four most parsimonious trees generated were with a tree length of 243 steps $(\mathrm{CI}=0.8807, \mathrm{RI}=0.8380, \mathrm{HI}=0.1193, \mathrm{RC}=$ $0.7380)$. ML analysis produced a tree with log likelihood (In) of $-2,281.445825$ (Fig. 3).

Based on phylogenetic analysis of the combined sequence of cox3 and nad 1 , the Verticillium isolates from horseradish were divided into three clades. All 10 isolates (CAV1003, CAV1004, CBV1006, CBV1014, CBV1046, SCV1005, SCV1010, SDV1002, SDV1006, and SDV1025) identified as V. dahliae based on morphological characteristics, and the reference strains of $V$. dahliae (76, M5, and UZ132) grouped together and formed the $V$. dahliae clade with moderate bootstrap support (67\% ML BP and 52\% MP BP). Ten isolates identified as $V$. longisporum based on morphological characteristics were divided into two clades in this tree. One isolate (CBV2034) from Ontario and four isolates (SCV2008, SDV2041, SDV2064, and SDV2066) from Illinois were grouped together (group B) and formed a distinct clade ( $94 \%$ ML BP, $<50 \%$ MP BP). This clade formed a sister group with the $V$. dahliae clade supported by moderately strong ML BP values, which means that this group was closely related to $V$. dahliae. Five isolates (TAV3001, TBV3014, VAV3003, VAV3021, and VBV3004) from California, identified as V. longisporum, and reference strains of V. longisporum (G19, $\mathrm{K} 2$, and $\mathrm{K} 12$ ) were grouped together and formed a clade with strong bootstrap support (100\% ML BP and 75\% MP BP). Interestingly, reference strains of $V$. longisporum $\mathrm{K} 2$ and $\mathrm{K} 12$, which grouped with $V$. longisporum group B in the ITS tree, were clustered together with group $\mathrm{A}$ in this tree. The five isolates in this clade (group A) showed a closer relationship (75\% ML BP, and $67 \%$ MP BP) to $V$. albo-atrum than to $V$. dahliae, although they were clearly distinct from both species (Fig. 3).

Pathogenicity test. All of the Verticillium isolates tested caused internal discoloration of roots. Symptoms of internal root discoloration developed over a period of 4 months in inoculated plants (Table 4). After 1 month, 33.3 and $16.7 \%$ of horseradish 1573 and BTW plants inoculated with $V$. longisporum group B isolates developed internal root discoloration. Both the incidence and severity of root discoloration increased gradually and, after 4 months, 83 to $100 \%$ of inoculated roots exhibited symptoms of internal discoloration. There were no significant differences in either the incidence or severity of internal discoloration of roots inoculated with $V$. dahliae and the two genetic groups (group A and B) of $V$. longisporum after 4 months from inoculation (Table 4). Also, there was no significant difference in either the incidence or severity of root discoloration between 1573 and BTW horseradish. The same Verticillium spp. used to inoculate roots was reisolated from the discolored roots.

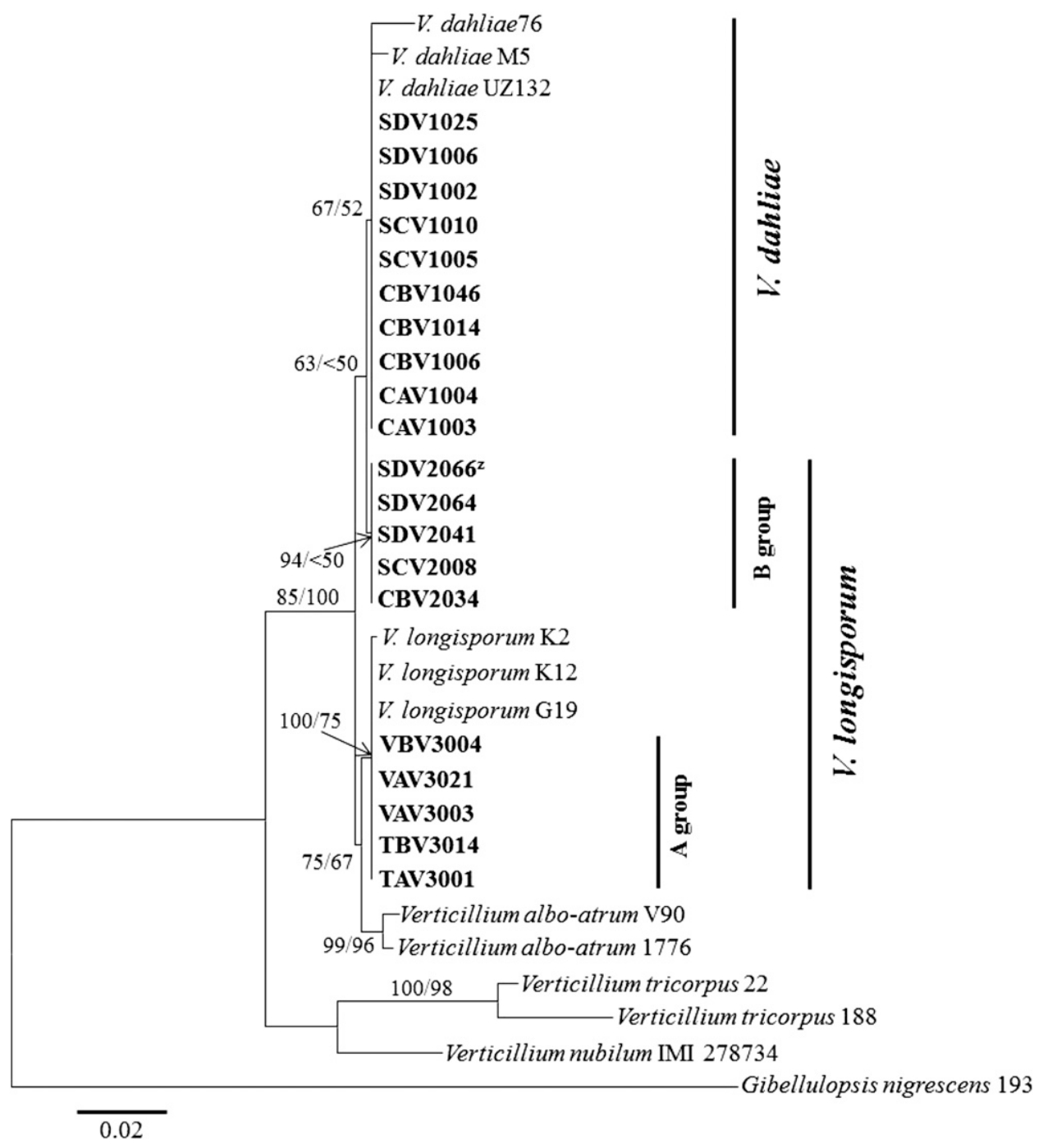

Fig. 3. Maximum-likelihood (ML) tree based on combined cytochrome oxidase subunit III and NADH dehydrogenase subunit I sequence data of the examined 20 isolates. ML and maximum-parsimony bootstrap values $\geq 50 \%$ are shown at nodes. Negative log likelihood of the $M L$ tree $=-2,281.445825$. Outgroup: Gibellulopsis nigrescens. Verticillium longisporum isolates in group A were from California, and V. Iongisporum isolates in group B and V. dahliae isolates were from Illinois and Ontario. V. Iongisporum isolate SDV-2066 $\left({ }^{\mathrm{Z}}\right)$ is the same isolate as reference strain V. Iongisporum PD730, which also was reported by Inderbitzin et al. (2011b). 
Control plants did not develop any symptoms and no pathogen was isolated from them.

\section{Discussion}

Conidial size is an important characteristic used to separate $V$. dahliae and V. longisporum (Hawksworth and Talboys 1970; Inderbitzin et al. 2011a; Karapapa et al. 1997; Stark 1961; Steventon et al. 2002). Other characteristics proposed by Karapapa et al. (1997) were microsclerotial morphology and number of phialides per node. They reported that $V$. dahliae produces relatively spherical microsclerotia and has 4 to 5 phialides on each conidiophore node, whereas $V$. longisporum produces elongate microsclerotia and usually has 3 phialides on each node. However, Inderbitzin et al. (2011a) reported that both $V$. dahliae and $V$. longisporum produce similar numbers of phialides in each node: 2 to 4 for $V$. dahliae and 2 to 5 for $V$. longisporum. In our study, we measured two different sizes of conidia but found similar numbers (2 to 4 ) of phialides per node for both $V$. dahliae and $V$. longisporum.

In the present study, conidial length of $V$. dahliae averaged $4.4 \mu \mathrm{m}$ $(<6.0 \mu \mathrm{m})$, while conidial length of $V$. longisporum averaged $7.8 \mu \mathrm{m}$ $(>6.5 \mu \mathrm{m})$, similar to the studies of Karapapa et al. (1997) and Steventon et al. (2002). Conidial lengths in our study were slightly shorter than that reported by Inderbitzin et al. (2011a). Most of microsclerotia of $V$. dahliae were rounded and measured 20 to $100 \mu \mathrm{m}$, while microsclerotia of $V$. longisporum were elongate to irregularly spherical and measured 30 to $250 \mu \mathrm{m}$. Thus, morphological characteristics of microsclerotia had some similarities, as reported by Inderbitzin et al. (2011a).

For phylogenetic analysis of Verticillium isolates, we used the ITS region (ITS1-5.8S-ITS2 region) and two mitochondrial genes, cox3 and nad 1 . The ITS region has been extensively used for phylogenetic analysis of Verticillium spp. (Fahleson et al. 2004; Inderbitzin et al. 2011b; Pantou et al. 2005) and other fungi (Suga et al. 2000). Mitochondrial genes cox3 and nad 1 have been used in phylogenetic studies of Verticillium isolates (Pantou et al. 2005) to differentiate species of Verticillium from each other, including V. dahliae and V. longisporum. In this study, using ITS1-5.8S-ITS2 did not differentiate $V$. dahliae from $V$. longisporum. When assessing the ITS tree, isolates of Verticillium from horseradish were divided into two clades: $V$. dahliae clade and V. longisporum clade. However, five isolates of $V$. longisporum from California (identified based on the morphological characteristics) and two reference strains of $V$. longisporum (G19 and PD687) clustered with $V$. dahliae. Similar results were also obtained by Pantou et al. (2005) and Inderbitzin et al. (2011a). According to Inderbitzin et al. (2011b), ITS alleles of $V$. longisporum isolates can be divided into two groups in correlation with the $V$. longisporum lineages D1, D2, and D3. Isolates of $V$. longisporum lineages D1 and D2 were shown to have identical ITS alleles and formed a sister group with $V$. dahliae, while ITS alleles of $V$. longisporum lineage $\mathrm{D} 3$ were identical to those of the majority of $V$. dahliae isolates. In this study, $V$. longisporum group A, which clustered to the $V$. dahliae clade, corresponded to V. longisporum lineage D3 of Inderbitzin et al. (2011b).

Several investigators have suggested that $V$. longisporum is an interspecific hybrid whose parents include V. dahliae, V. albo-atrum, or close relatives of these two species (Clewes et al. 2008; ColladoRomero et al. 2010; Collins et al. 2003; Karapapa et al. 1997), and their genetic diversities have been characterized (Barbara and Clewes 2003; Collins et al. 2003, 2005; Karapapa et al. 1997; Pantou et al. 2005; Zeise and von Tiedemann 2001). Previously, Inderbitzin et al. (2011b) conducted phylogenetic analyses of seven nuclear loci and a dataset of 203 isolates of $V$. dahliae, $V$. longisporum, and related species. They suggested that $V$. longisporum is an allodiploid hybrid that originated three different times through independent hybridization events, involving four different parental lineages and three different species. Also, according to Inderbitzin et al. (2011b), all of the hybrids share a common parent, species A1, that hybridized separately with species $\mathrm{D} 1, V$. dahliae lineage $\mathrm{D} 2$, and $V$. dahliae lineage $\mathrm{D} 3$, giving rise to the three different lineages, including $V$. longisporum lineage A1/D1, $V$. longisporum lineage A1/D2, and $V$. longisporum lineage A1/D3, respectively. Another study at the same time (Inderbitzin et al. 2011a) suggested that $V$. longisporum lineage A1/D1 corresponds to Zeise and von Tiedemann's group 1sp (Zeise and von Tiedemann 2001) and Clewes et al. (2008) group $\alpha$, whereas V. longisporum lineage A1/D2 corresponds to group $\beta \gamma$ of Clewes et al. (2008). Also, the $V$. longisporum lineage A1/D3 corresponds to group 1sp of Zeise and von Tiedemann (2001) and group $\beta$ of Clewes et al. (2008).

In our study, we obtained two genetic groups (group A and group B) of $V$. longisporum based on phylogenetic analysis of the ITS15.8S-ITS2 region as well as the combined sequence of $\operatorname{cox} 3$ and nad1. These two groups of $V$. longisporum were similar to the two different lineages of $V$. longisporum reported by Inderbitzin et al. (2011b). The $V$. longisporum genetic groups A and B corresponded to $V$. longisporum lineages A1/D3 and A1/D2, respectively, of Inderbitzin et al. (2011b). Furthermore, V. longisporum group A of our isolates and $V$. longisporum lineage A1/D3 of Inderbitzin et al. (2011b) clustered together with the $V$. dahliae clade in the ITS tree. $V$. longisporum reference strains $\mathrm{K} 2$ and $\mathrm{K} 12$ clustered with group B in the ITS tree, even though they clustered into group A in the combined cox 3 and nad 1 tree. Phylogenetic analysis of the combined sequence of the ITS region, cox3, and nad 1 showed that strains $\mathrm{K} 2$ and K12 formed a distinct clade (data not shown). According to the results, we concluded that $V$. longisporum reference strains $\mathrm{K} 2$ and $\mathrm{K} 12$

Table 4. Occurrence of internal root discoloration caused by artificial inoculation of Verticillium dahliae and V. longisporum on horseradish '1573' and 'Big Top Western'

\begin{tabular}{|c|c|c|c|c|c|c|c|c|c|c|c|}
\hline \multirow[b]{3}{*}{ Treatment $^{\mathrm{y}}$} & \multirow[b]{3}{*}{ Occurrence $^{\mathrm{z}}$} & \multicolumn{10}{|c|}{ Disease development in months ${ }^{x}$} \\
\hline & & \multicolumn{5}{|c|}{1573} & \multicolumn{5}{|c|}{ Big Top Western } \\
\hline & & 1 & 2 & 3 & 4 & $\overline{\text { LSD }}$ & 1 & 2 & 3 & 4 & $\overline{\text { LSD }}$ \\
\hline \multirow{2}{*}{$\overline{V . \text { dahliae }}$} & Incidence & $0 \mathrm{a}$ & $50.0 \mathrm{~b}$ & $83.3 \mathrm{bc}$ & $100.0 \mathrm{c}$ & 41.4 & $0 \mathrm{a}$ & $50.0 \mathrm{~b}$ & $83.3 \mathrm{~b}$ & $83.3 \mathrm{~b}$ & 47.9 \\
\hline & Severity & $0 \mathrm{a}$ & $1.8 \mathrm{ab}$ & $21.7 \mathrm{bc}$ & $37.6 \mathrm{c}$ & 16.3 & $0 \mathrm{a}$ & $10.2 \mathrm{a}$ & $18.3 \mathrm{a}$ & $37.9 \mathrm{~b}$ & 18.6 \\
\hline \multirow{2}{*}{ V. longisporum (group A) } & Incidence & $0 \mathrm{a}$ & $33.3 \mathrm{a}$ & $83.3 \mathrm{~b}$ & $100 \mathrm{~b}$ & 39.6 & $0 \mathrm{a}$ & $33.3 \mathrm{a}$ & $83.3 \mathrm{~b}$ & $100.0 \mathrm{~b}$ & 39.6 \\
\hline & Severity & $0 \mathrm{a}$ & $6.0 \mathrm{a}$ & $21.7 \mathrm{~b}$ & $31.3 \mathrm{~b}$ & 12.1 & $0 \mathrm{a}$ & $3.8 \mathrm{ab}$ & $18.3 \mathrm{~b}$ & $37.6 \mathrm{c}$ & 15.2 \\
\hline \multirow[t]{2}{*}{ V. longisporum (group B) } & Incidence & $33.3 \mathrm{a}$ & $66.7 \mathrm{ab}$ & $83.3 \mathrm{ab}$ & $100.0 \mathrm{~b}$ & 50.4 & $16.7 \mathrm{a}$ & $50.0 \mathrm{ab}$ & $66.7 \mathrm{ab}$ & $100.0 \mathrm{~b}$ & 51.6 \\
\hline & Severity & $1.7 \mathrm{a}$ & $5.5 \mathrm{a}$ & $18.3 \mathrm{~b}$ & $34.7 \mathrm{c}$ & 9.7 & $0.8 \mathrm{a}$ & $2.5 \mathrm{a}$ & $25.3 \mathrm{~b}$ & $28.0 \mathrm{~b}$ & 13.7 \\
\hline \multirow[t]{2}{*}{ Control } & Incidence & 0 & 0 & 0 & 0 & NS & 0 & 0 & 0 & 0 & NS \\
\hline & Severity & 0 & 0 & 0 & 0 & NS & 0 & 0 & 0 & 0 & NS \\
\hline
\end{tabular}

x Values within each row and for each cultivar with a letter in common are not significantly different from each other according to Fisher's protected least significant difference (LSD; $P=0.05)$. NS = not significant.

y $V$. dahliae isolates from Ontario (CBV 1014 isolate), V. longisporum isolates from California (group A, TAV3001 isolate), and V. longisporum isolates from Illinois (group B, SCV2008 isolate).

${ }^{\mathrm{z}}$ Disease occurrence. Incidence $=$ percentage of plants with discolored root. Data represent the average of two experiments, each with 24 plants. Severity $=$ percent root area at the cross-section discolored. Severity of discoloration was based on a 0 -to-4 scale, where $0=0,1=1$ to 10 (average 5 ), $2=11$ to 25 (17.5), $3=26$ to $50(37.5)$, and $4=51$ to $100 \%(75 \%)$. 
from oilseed rape belong to another separate lineage. In this study, we could not isolate the third group corresponding to $V$. longisporum lineage A1/D1 of Inderbitzin et al. (2011b) from horseradish.

Inderbitzin et al. (2011b) reported that $V$. longisporum lineage A1/D2 was confined to horseradish in Illinois, whereas lineage A1/D3 was restricted to Europe and Japan, and lineage A1/D1 occurred in Europe, Japan, and North America. In this study, we found that $V$. longisporum lineage A1/D2 (group B in this study) occurred not only in Illinois but also in Ontario, whereas lineage A1/D3 (group A in this study) occurred only in California. The predominant species of Verticillium varied according to the horseradish growing area. Specifically, $V$. dahliae was the most prevalent in root samples from Illinois and Ontario, whereas $V$. longisporum group A (lineage A1/D3 of Inderbitzin et al. [2011b]) was the most prevalent in root samples from California.

Both $V$. dahliae and $V$. longisporum have been isolated from oilseed rape but $V$. longisporum was determined to be the cause of Verticillium wilt in oilseed rape (Eynck et al. 2007; Heale and Karapapa 1999). Subbarao et al. (1995) reported that $V$. longisporum caused stunting, chlorosis, and discoloration of vessels in leaves in oilseed rape. Novakazi et al. (2015) conducted comparative pathogenicity tests using $V$. dahliae and three $V$. longisporum lineages on 11 different hosts, including artichoke, cabbage, cauliflower, cotton, eggplant, horseradish, lettuce, linseed, oilseed rape, tomato, and watermelon. This study demonstrated that $V$. longisporum was more virulent than $V$. dahliae on brassica hosts, including horseradish. They also showed that virulence of each $V$. longisporum lineage varied with host. For example $V$. longisporum A1/D2 was the most virulent group on horseradish and cabbage, whereas $V$. longisporum A1/D1 showed higher virulence than $V$. dahliae on oilseed rape and cauliflower (Novakazi et al. 2015). V. longisporum A1/D3 was not highly virulent, and $V$. dahliae was less virulent than three $V$. longisporum lineages tested on all of the hosts they evaluated. In our study, pathogenicity tests revealed that both $V$. dahliae and two identified genetic groups of $V$. longisporum were pathogenic to horseradish and caused internal discoloration of roots. However, we did not establish species-specific symptom characteristics of our isolates. The roots inoculated with $V$. longisporum group A (corresponding to $V$. longisporum lineage A1/D2) showed disease symptoms earlier (1 month after inoculation) than other Verticillium groups, and showed higher disease incidence at 2 months after inoculation. However, there were no significant differences in either the incidence or severity of root discoloration among isolates of $V$. dahliae and two genetic groups of $V$. longisporum 4 months after inoculation. This finding explains that $V$. longisporum lineage A1/D2 was more virulent than other groups in the pathogenicity tests conducted for 9 weeks by Novakazi et al. (2015).

International root discoloration of horseradish is a disease complex. This study provided additional information about the pathogens causing the internal root discoloration of horseradish. However, for developing strategies for effective management of the disease, further studies of the etiology of the disease are needed.

\section{Acknowledgments}

This research was supported, in part, by funds from the North Central Integrated Pest Management Program. We thank K. Traaseth, S. Sius, R. Marshal, and W. Vanderstelt for providing horseradish roots; and M. S. Park, J. X. Deng, and H. Sang for the technical assistance of phylogenetic analysis.

\section{Literature Cited}

Atibalentja, N., and Eastburn, D. 1998. Verticillium dahliae resistance in horseradish germ plasm from the University of Illinois collection. Plant Dis. 82:176-180.

Babadoost, M., Chen, W., Bratsch, A., and Eastman, C. 2004. Verticillium longisporum and Fusarium solani: Two new species in the complex of internal discoloration of horseradish roots. Plant Pathol. 53:669-676.

Barbara, D. J., and Clewes, E. 2003. Plant pathogenic Verticillium species: How many of them are there? Mol. Plant Pathol. 4:297-305.

Boning, K. 1938. Die wichtigsten Krankheiten und Schädlinge des meerrettichs. [The most important diseases and pests of horseradish] Rev. Appl. Mycol. 13:62-87.

Carder, J., and Barbara, D. 1994. Molecular variation within some Japanese isolates of Verticillium dahliae. Plant Pathol. 43:947-950.
Clewes, E., Edwards, S., and Barbara, D. J. 2008. Direct molecular evidence supports long-spored microsclerotial isolates of Verticillium from crucifers being interspecific hybrids. Plant Pathol. 57:1047-1057.

Collado-Romero, M., Jiménez-Díaz, R. M., and Mercado-Blanco, J. 2010. DNA sequence analysis of conserved genes reveals hybridization events that increase genetic diversity in Verticillium dahliae. Fungal Biol. 114:209-218.

Collins, A., Mercado-Blanco, J., Jiménez-Díaz, R. M., Olivares, C., Clewes, E. and Barbara, D. 2005. Correlation of molecular markers and biological properties in Verticillium dahliae and the possible origins of some isolates. Plant Pathol. 54:549-557.

Collins, A., Okoli, C. A. N., Morton, A., Parry, D., Edwards, S. G., and Barbara, D. J. 2003. Isolates of Verticillium dahliae pathogenic to crucifers are of at least three distinct molecular types. Phytopathology 93:364-376.

Courter, J., and Rhodes, A. 1969. Historical notes on horseradish. Econ. Bot. 23: 156-164.

Eastburn, D., and Chang, R. 1994. Verticillium dahliae: A causal agent of root discoloration of horseradish in Illinois. Plant Dis. 78:496-498.

Eranthodi, A., Babadoost, M., and Trierweiler, B. 2010. Thermotherapy for control of fungal pathogens in propagative rootstocks of horseradish. HortScience 45: 599-604.

Eynck, C., Koopmann, B., Grunewaldt-Stoecker, G., Karlovsky, P., and Von Tiedemann, A. 2007. Differential interactions of Verticillium longisporum and $V$. dahliae with Brassica napus detected with molecular and histological techniques. Eur. J. Plant Pathol. 118:259-274.

Fahleson, J., Hu, Q., and Dixelius, C. 2004. Phylogenetic analysis of Verticillium species based on nuclear and mitochondrial sequences. Arch. Microbiol. 181: 435-442.

Hall, T. A. 1999. BioEdit: A user-friendly biological sequence alignment editor and analysis program for Windows 95/98/NT. Nucleic Acids Symp. Ser. 41: 95-98.

Hawksworth, D. L., and Talboys, P. W. 1970. Verticillium dahliae. CMI Descr Pathogenic Fungi Bact. No. 256. Commonwealth Mycological Institute, Kew, Surrey, England.

Heale, J. B., and Karapapa, V. K. 1999. The Verticillium threat to Canada's major oilseed crop. Canola. Can. J. Plant Pathol. 21:1-7.

Inderbitzin, P., Bostock, R. M., Davis, R. M., Usami, T., Platt, H. W., and Subbarao, K. V. 2011a. Phylogenetics and taxonomy of the fungal vascular wilt pathogen Verticillium, with the descriptions of five new species. PLoS One 6:e28341

Inderbitzin, P., Davis, R. M., Bostock, R. M., and Subbarao, K. V. 2011b. The ascomycete Verticillium longisporum is a hybrid and a plant pathogen with an expanded host range. PLoS One 6:e18260.

Isaac, I. 1957. Verticillium wilt of Brussels sprout. Ann. Appl. Biol. 45:276-283.

Jeon, Y. S., Chung, H., Park, S., Hur, I., Lee, J. H., and Chun, J. 2005. jPHYDIT: A Java-based integrated environment for molecular phylogeny of ribosomal RNA sequences. Bioinformatics 21:3171-3173.

Karapapa, V., Bainbridge, B., and Heale, J. 1997. Morphological and molecular characterization of Verticillium longisporum comb. nov., pathogenic to oilseed rape. Mycol. Res. 101:1281-1294.

Klosterman, S. J., Atallah, Z. K., Vallad, G. E., and Subbarao, K. V. 2009 Diversity, pathogenicity, and management of Verticillium species. Annu. Rev. Phytopathol. 47:39-62.

Koike, S. T., Subbarao, K. V., Davis, R. M., Gordon, T. R., and Hubbard, J. C 1994. Verticillium wilt of cauliflower in California. Plant Dis. 78:1116-1121.

Korff, G., and Boning, K. 1934. Die Meerrettichschwarse und ihre Bekampfung. Prakt. Bl. Pflanzenschutz 11:9-10.

Kouvelis, V. N., Ghikas, D. V., and Typas, M. A. 2004. The analysis of the complete mitochondrial genome of Lecanicillium muscarium (synonym Verticillium lecanii) suggests a minimum common gene organization in mtDNAs of Sordariomycetes: Phylogenetic implications. Fungal Genet. Biol. 41:930-940.

Mueller, J., Percich, J., and Mitchell, J. 1982. Root deterioration associated with Verticillium wilt of horseradish. Plant Dis. 66:410-414.

Novakazi, F., Inderbitzin, P., Sandoya, G., Hayes, R. J., von Tiedemann, A., and Subbarao, K. V. 2015. The three lineages of the diploid hybrid Verticillium longisporum differ in virulence and pathogenicity. Phytopathology 105: 662-673.

Pantou, M. P., Mavridou, A., and Typas, M. A. 2003. IGS sequence variation, group-I introns and the complete nuclear ribosomal DNA of the entomopathogenic fungus Metarhizium: Excellent tools for isolate detection and phylogenetic analysis. Fungal Genet. Biol. 38:159-174.

Pantou, M. P., Strunnikova, O. K., Shakhnazarova, V. Y., Vishnevskaya, N. A., Papalouka, V. G., and Typas, M. A. 2005. Molecular and immunochemical phylogeny of Verticillium species. Mycol. Res. 109:889-902.

Percich, J., and Johnson, D. 1990. A root rot complex of horseradish. Plant Dis. 74: 391-393.

Potschke, A. 1923. Über das Scharzwerden des Meerrettichs. [On the black discoloration of horseradish]. Biol. Reichsanst. Land Forst-wirtsch. Arb. 11: 337-338.

Rhodes, A. 1977. Horseradish problems and research in Illinois. Pages 137-148 in: Crop Resources. D. S. Seigler, ed. Academic Press, Inc., New York.

Sorauer, P. 1899. Kernfaule unt Schwarzwerden des Meerrettich. Z. Pflanzenkrankh. 9:132-137. 
Stamatakis, A. 2006. RAxML-VI-HPC: Maximum likelihood-based phylogenetic analyses with thousands of taxa and mixed models. Bioinformatics 22:2688-2690.

Stamatakis, A., Hoover, P., and Rougemont, J. 2008. A rapid bootstrap algorithm for the RAxML web servers. Syst. Biol. 57:758-771.

Stark, C. 1961. Das Auftreten der Verticillium-Tracheomykosen in Hamburger Gartenbaukulturen. Gartenbauwissenschaft 26:493-528.

Steventon, L. A., Fahleson, J., Hu, Q., and Dixelius, C. 2002. Identification of the causal agent of Verticillium wilt of winter oilseed rape in Sweden, V. longisporum. Mycol. Res. 106:570-578.

Subbarao, K., Chassot, A., Gordon, T., Hubbard, J., Bonello, P., Mullin, R., Okamoto, D., Davis, R., and Koike, S. 1995. Genetic relationships and cross pathogenicities of Verticillium dahliae isolates from cauliflower and other crops. Phytopathology 85:1105-1112.

Suga, H., Hasegawa, T., Mitsui, H., Kageyama, K., and Hyakumachi, M. 2000. Phylogenetic analysis of the phytopathogenic fungus Fusarium solani based on the rDNA-ITS region. Mycol. Res. 104:1175-1183.

Svensson, C. H., and Lerenius, C. 1987. An investigation on the effect of Verticillium wilt (Verticillium dahliae Kleb.) on oilseed rape. Working group integrated control in oilseed rape. IOBC WPRS Bull. 4:30-34.
Swofford, D. 1998. PAUP 4.0: Phylogenetic Analysis Using Parsimony. Smithsonian Institution, Washington, DC.

Tamura, K., Peterson, D., Peterson, N., Stecher, G., Nei, M., and Kumar, S. 2011 MEGA5: Molecular evolutionary genetics analysis using maximum likelihood, evolutionary distance, and maximum parsimony methods. Mol. Biol. Evol. 28:2731-2739.

Thompson, J. D., Gibson, T. J., Plewniak, F., Jeanmougin, F., and Higgins, D. G. 1997. The CLUSTAL_X windows interface: Flexible strategies for multiple sequence alignment aided by quality analysis tools. Nucleic Acids Res. 25:4876-4882

White, T. J., Bruns, T., Lee, S., and Taylor, J. 1990. Amplification and direct sequencing of fungal ribosomal RNA genes for phylogenetics. Pages 315322 in: PCR Protocols: A Guide to Methods and Applications. Innis, M. A., Gelfand, D. H., Sninsky, J. J., and White, T. J., eds. Academic Press, San Diego, CA.

Yu, J. M., and Babadoost, M. 2013. Occurrence of Fusarium commune and F. oxysporum in Horseradish Roots. Plant Dis. 97:453-460.

Zeise, K., and von Tiedemann, A. 2001. Morphological and physiological differentiation among vegetative compatibility groups of Verticillium dahliae in relation to $V$. longisporum. J. Phytopathol. 149:469-475. 